\title{
On Modeling of Ultrathin Field Emitter
}

\author{
V.A.Fedirko ${ }^{1,3, *}$, S.V. Polyakov ${ }^{2,3}$ \\ ${ }^{1}$ Moscow State University of Technology "Stankin" (MGTU “Stankin"), Moscow, Russia \\ ${ }^{2}$ Keldysh Institute of Applied Mathematics, Moscow, Russia \\ ${ }^{3}$ National Research University of Electronic Technology "MIET" (MIET), Moscow, Russia \\ *Corresponding Author: vfed@mail.ru
}

Copyright (C) 2014 Horizon Research Publishing All rights reserved.

\begin{abstract}
An analytical model for the potential barrier near the apex of an ultrathin edge field emitter is suggested. It is used for the numerical modeling of electron tunneling from the emitter. The results show that the conventional approximation of a uniform field near the edge is not proper for an ultrathin emitter. The suggested approach is more adequate for modeling of field emission from nano-sized cold cathodes.
\end{abstract}

Keywords Electron Tunneling, Potential Barrier, Field Emission, Nano-Sized Cold Cathode, Conformal Transformation, Transfer Matrix Method

\section{Introduction}

Electron field emission is an extremely appealing quantum phenomena in view of its high potential in vacuum micro/nano-electronic applications [1]. Cold field emission cathodes are being considered also as the electron sources for propellant ionization and ion beam neutralization in electric propulsion systems [2]. Field emission results from quantum electron tunneling in high enough electric field which is usually achieved by forming an arrow-headed cathode. The electrostatic field enhancement factor $\beta$ increases with aspect ratio $h / r$, where $h$ is the height of an emitter and $r$ is its apex radius. The more the $\beta$ the more the emitting current, and that stimulates the tendency to form emitters with higher aspect ratio. For that reason there has been an increasing interest lately to field emission study from nanostructures due to the significant progress in nanotechnology. 2D and 1D nanomaterials have been extensively studied in view of their use as field emitters for electron beam lithography, field emission flat displays, $\mathrm{x}$-ray sources, sensors and microwave devices [3-7]. And recently graphene has attracted interest as potential candidate for effective electron field emitters with rather low turn-on voltage and high emission current [8-10].

As a rule, the estimation of the emitting current when analyzing the experimental data is based on the conventional Fauler-Nordheim formula [11]. That approach may however fail when $r$ is very small. In that case the electric field near the tip of the emitter is extremely non-uniform which gets principal for adequate calculation of the tunneling probability. One should also take into account that image potential also needs some modification as compared to well-known Shottky formula.

In this paper we present an analytical model for the potential barrier near the apex of an ultrathin edge field emitter in high electric field and results of numerical calculations for tunneling probability which demonstrates the effect of non-uniform electric field near the top of such an emitter. The results are relevant for nano-sized cold cathode structures, including graphene field emitters.

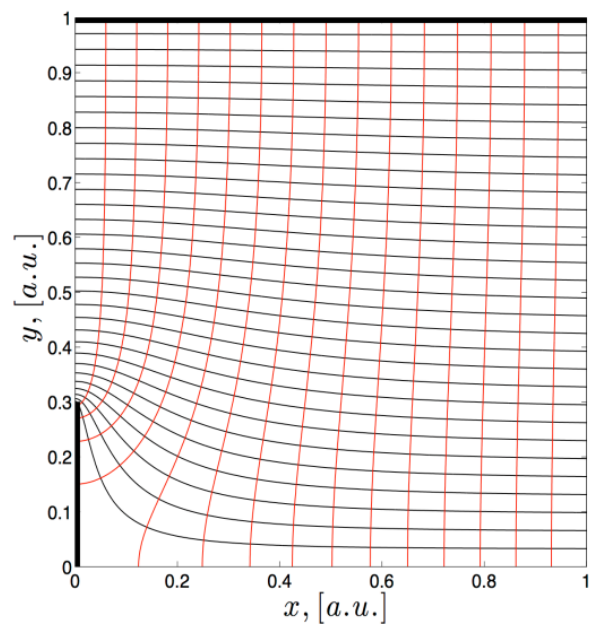

Figure 1. Contour lines for $w(z)$ of $(1)$ : black $-\operatorname{Im} w(z)=$ const; red $-\operatorname{Re} w=$ const

\section{Diode Structure}

The electrostatic potential in 2D geometry can be found using conformal transformation technique for the corresponding Dirihle problem. For a plane diode structure the required transformation is [13]:

$$
w(z)=\frac{2}{\pi} \operatorname{arth}\left(\sqrt{\cos ^{2} \frac{\pi \eta}{2} \cdot\left(\operatorname{th}^{2} \frac{\pi z}{2}+\operatorname{tg}^{2} \frac{\pi \eta}{2}\right)}\right),
$$


where $z=x+i y$, coordinates $x, y$ are normalized by the distance between the anode and the cathode $H, \eta=h / H$. The 2D potential distribution, $\varphi(x, y)$, normalized by anode potential $V$, is given by $\varphi(x, y)=\operatorname{Im} w$. The contour lines for the $\operatorname{Re} w$ and $\operatorname{Im} w$, calculated for a structure with $\eta=0.3$, are shown as an example in Fig. 1.

The electric field is found as a derivative of $w^{*}(z)-$ complex conjugate to $w(z)$ :

$$
E=E_{x}+i E_{y}=-i \frac{d w^{*}}{d z} .
$$

On the apex one has:

$$
E_{x}=0 ; E_{y}=\frac{\sin \frac{\pi(\eta+\rho)}{2}}{\sqrt{\sin \pi\left(\eta+\frac{\rho}{2}\right) \sin \frac{\pi \rho}{2}}},
$$

where $\rho=r / H$. When $\rho<<\eta$ it gives:

$$
E_{y} \approx-\sqrt{\frac{\operatorname{tg} \frac{\pi \eta}{2}}{\pi \rho}} ;
$$

and if in turn $\eta<<1$ it results in

$$
E_{y} \approx-\sqrt{\frac{\eta}{2 \rho}},
$$

or in dimensional variables:

$$
E_{y} \approx-\frac{V}{H} \sqrt{\frac{h}{2 r}},
$$

$V$ being the anode voltage. The field enhancement factor on the tip of an emitter is:

$$
\beta=\sqrt{\frac{h}{2 r}} .
$$

In common, while the tunneling probability is calculated, the normal component of the electric field near the tip of the emitter is supposed uniform and equal to its value on the apex. For an emitter with ultra-thin emitter, as can be seen from (1), (2), this is no longer a proper approximation. Besides, more refined formula for image potential must be used, like that for a charge near a conducting half-plane (see, e. g., [14]):

$$
U_{i}(x, y)=-\frac{e^{2}}{16 \pi^{2} \varepsilon_{0} d}\left[1+\frac{\pi-\theta}{\sin \theta}\right],
$$

where $\theta$ is the angle between the plane and the direction from the edge to the point charge, $d$ is the distance between them. As a result the normalized potential barrier at the edge of the emitter along the normal to it $(\theta=\pi)$ takes the form:

$$
U(y)=1-\frac{e V}{U_{0}}[\phi(0, y)-\phi(0, \eta+\rho)]-\frac{b}{y-\eta-\rho+b},
$$

where we use the intrinsic barrier height of the emitter $U_{0}$ for normalization, and choose parameter $b$ to fit $U(y)=0$ at the surface of the emitter $y=\eta-\rho$ :

$$
b=\frac{e^{2}}{8 \pi^{2} \varepsilon_{0} U_{0} H} .
$$

Fig. 2 shows the resulting barrier (5) in comparison with conventional "uniform field" barrier calculated for $\mathrm{H}=1 \mathrm{~mm}$, $\mathrm{h}=1 \mu, \mathrm{r}=1,33 \AA, \mathrm{U} 0=4,5 \mathrm{eV}, \mathrm{V}=100 \mathrm{kV}$. One can clearly see that the barriers are markedly different.

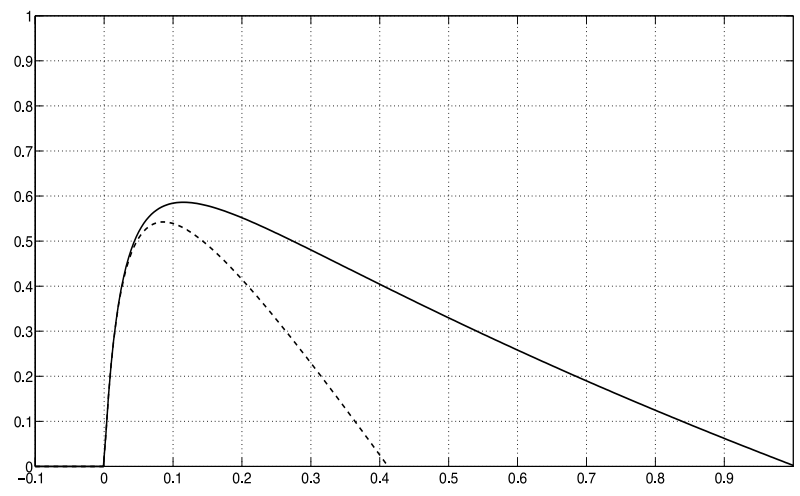

Figure 2. Potential barriers at the edge of the emitter in a diode structure with $H=1 \mathrm{~mm}, h=1 \mu, r=1.33 \AA, U_{0}=4.5 \mathrm{eV}, V=100 \mathrm{kV}$ : solid line - formula (5), dashed line - "uniform field" approximation A large figure in one column

The reason for that is quite apparent. The "uniform field" approximation is good enough at a distance so small that the surface of a tip still "looks flat" - that is true for $[y-(\eta+\rho)]<<\rho$ and corresponds to the first term in Taylor series of $\varphi(y)$ in $\left[y-\left(\eta^{+} \rho\right)\right] / \rho$. It obviously fails when $[y-(\eta+\rho)] \geq \rho$. For the case of fig. 2 the barrier width exceeds the tip radius, that's why the linear approximation for $\varphi(y)$ becomes inadequate. As the tunneling probability is determined by the height and the width of a potential barrier the constant field approximation thus will result in noticeable errors in estimation of the emission current.

To estimate the above mentioned difference of the tunneling transparency we have solved numerically 1D Schrödinger equation for both barriers using transfer matrix technique $[15,16]$. The results are shown in fig 3 . One can see that the uniform (constant) field approximation indeed overestimates the tunneling probability particularly for low energies which are most actual for field emission. That is also quite understandable: the difference between the effective widths of the two barriers is more for low energies, as seen from fig. 2, while the heights of the barriers differs slightly.

In fig. 4 we present for comparison the same curves calculated for a diode structure wit the parameters: $H=1 \mathrm{mM}$, $h=1 \mu, r=0.5 \mathrm{~nm}, U_{0}=4,5 \mathrm{eV}, V=100 \mathrm{kV}$. The estimations of tunneling coefficient for the two models still differ noticeably in that case too. 


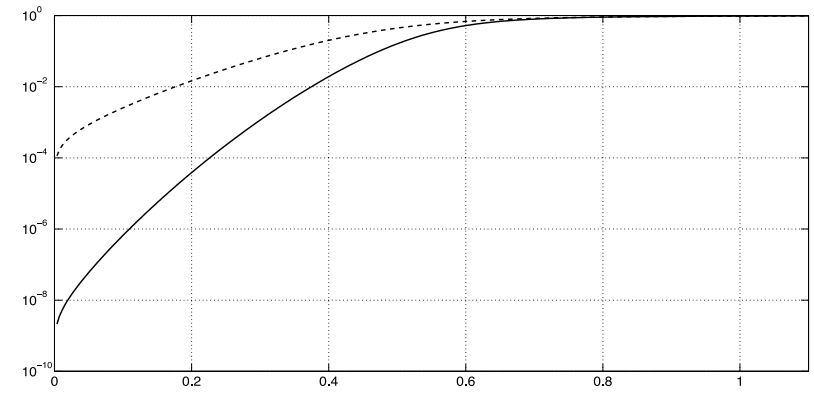

Figure 3. Tunneling probability vs. energy for a diode structure with $H$ $=1 \mathrm{~mm}, h=1 \mu, r=1.33 \AA, U_{0}=4.5 \mathrm{eV}, V=100 \mathrm{kV}$ : solid line - formula (5), dashed line - "uniform field" approximation A large figure in one column

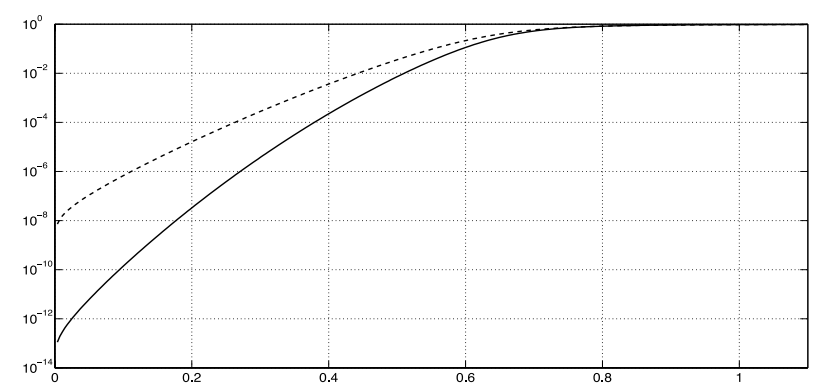

Figure 4. Tunneling probability vs. energy for a diode structure with $H$ $=1 \mathrm{~mm}, h=1 \mu, r=0.5 \mathrm{~nm}, U_{0}=4.5 \mathrm{eV}, V=100 \mathrm{kV}$ : solid line - barrier (5), dashed line - the "uniform field" approximation A large figure in one column

\section{Slit-grid Structure}

To form high electric field at the edge of an emitter a triode-like structure is used, with a grid having slits at the tip of the emitters. For that case it is also possible to solve the corresponding Dirihle problem with a proper conformal mapping $\mathrm{w}(z)$ in a complex plain $z=x+i y$ :

$$
w(z)=\frac{2}{\pi} \operatorname{acrth}\left(\sqrt{\left(z-\sqrt{z^{2}-1}\right)^{2}+1}\right) .
$$

The contour lines for the $\operatorname{Re} w$ and $\operatorname{Im} w$ are shown as an example in Fig. 5, Im $w=$ const being the equipotential curves.

The electric field at the top of the emitter, at $\eta=i \rho$ is (supposing $\rho<<\eta$ ):

$$
E_{y} \approx-\sqrt{\frac{2}{\pi^{2} \rho}},
$$

or

$$
E_{y} \approx-\frac{V}{\pi a} \sqrt{\frac{2 a}{r}}
$$

in dimensional variables as in (7) we normalized the coordinates by the distance $a$ between the cathode tip and the grid slit edge. And the field enhancement factor on the tip is then:

$$
\beta=\sqrt{\frac{2 a}{r}} .
$$

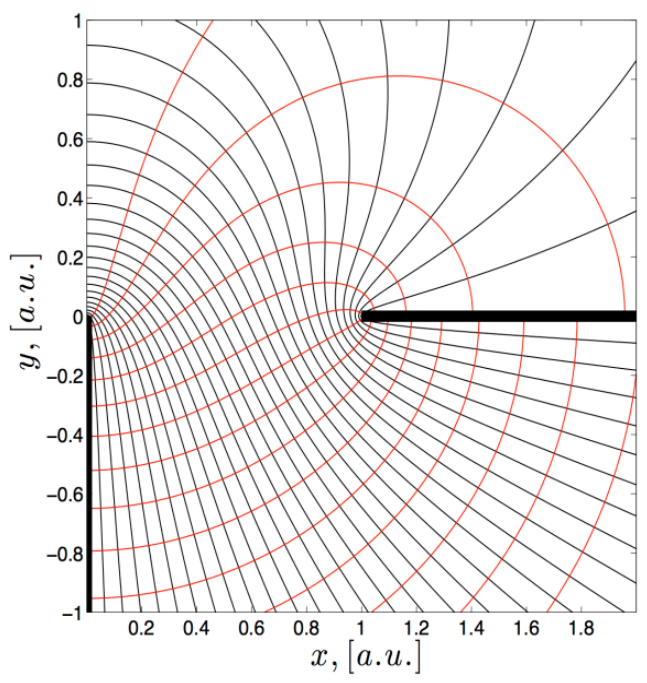

Figure 5. Contour lines for (6): black $-\operatorname{Im} w(z)=$ const ; red $-\operatorname{Re} w=$ const

The resulting barriers calculated for $a=0.1 \mu, r=1.33 \AA$, $U_{0}=4.5 \mathrm{eV}, V=50 \mathrm{~V}$ are presented in Fig. 6 , fig. 7 shows the calculated tunneling transparency for that barriers.

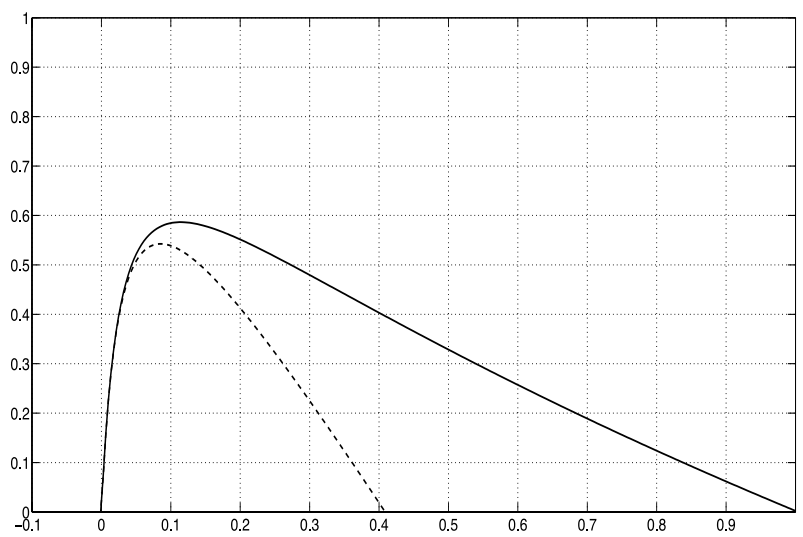

Figure 6. Potential barriers at the edge of the emitter in a slit-grid structure with $a=0.1 \mu, r=1.33 \AA, U_{0}=4.5 \mathrm{eV}, V=50 \mathrm{~V}$ : solid line - formula (5), dashed line - "uniform field" approximation

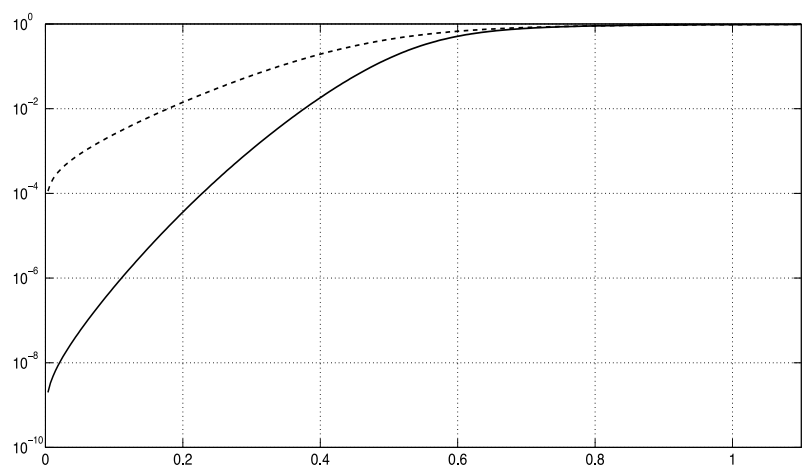

Figure 7. Tunneling probability vs. energy for a slit-grid structure with $a=0.1 \mu, r=1.33 \AA, U_{0}=4.5 \mathrm{eV}, V=50 \mathrm{~V}$ : solid line - barrier (5), dashed line the "uniform field" approximation 
For the slit-gate structure with $a=0.1 \mu, r=0.5 \mathrm{~nm}$, $U_{0}=4,5 \mathrm{eV}, \quad V=50 \mathrm{~V}$ the difference between tunneling coefficients calculated in the two models is also rather significant as seen in Fig. 8.

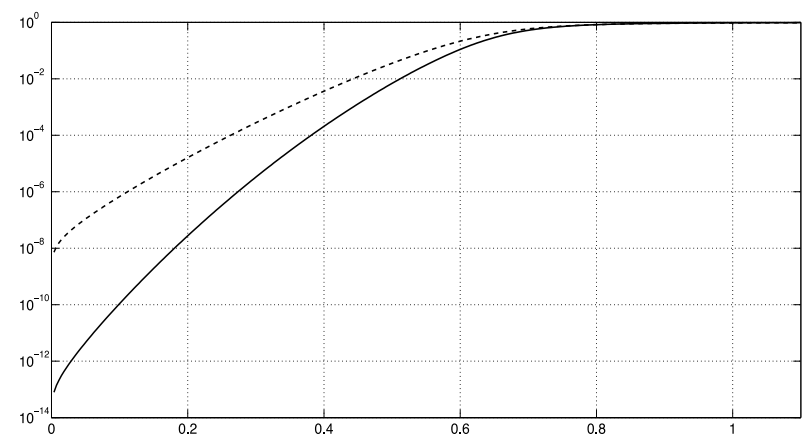

Figure 8. Tunneling probability vs. energy for a slit-grid structure with $a=0.1 \mu, r=0.5 \mathrm{~nm}, U_{0}=4.5 \mathrm{eV}, V=50 \mathrm{~V}$ : solid line - barrier (5), dashed line the "uniform field" approximation

\section{Conclusion}

The results of our modeling show that the widely used approximation of uniform electric field near the emitting surface for estimation of tunneling probability fails for very thin field emitter. The electric field in under-barrier region no longer can be treated as a constant when the effective barrier width is comparable to the tip radius. The effective barrier width turns out grater in this case as the "mean" field is actually less than its value on the top of an emitter. That's why the conventional uniform field approach results in overestimation of tunneling probability and to overestimation of the emitting current in the final analysis. So an inelaborate use of high apex ratio emitter will not lead to the expected enhancement of the emission current. It should be taken into account when designing auto-cathodes with ultra-thin or very pointy emitters and modeling their characteristics.

The above effect of mean field reduction can be avoided by using the emitter with aspect ratio so high as to implement the effective barrier narrower than the apex radius:

$$
L_{0} \approx \frac{U_{0} H}{e V} \sqrt{\frac{2 r}{h}} \leq r,
$$

For fixed values of $U_{0}, r, H / V$, it can be reached by enlargement the height of an emitter up to:

$$
h \geq \frac{2 U_{0}^{2} H^{2}}{r e^{2} V^{2}} .
$$

For $U_{0}=4.5 \mathrm{eV}, r \approx 1.33 \AA$ (about the thickness of graphene monolayer) and $H / V \approx 10^{6} \mathrm{~V} / \mathrm{cm}$ that gives $h \geq 30 \mu$. However it may be of some technological problem to make such free standing emitter.

In conclusion we suggest an analytical model for a potential barrier near the top of an ultra-thin edge field emitter which is more appropriate than the conventional approximation. It takes into account the non-uniformity of the electrical field in the under-barrier region and uses more accurate formula for the image potential. The model is applicable for more adequate modeling of quantum electron tunneling in such structures.

We certainly realize that 1D approach we used for estimation of the tunneling probability may be insufficient for tips of near-atomic size but that is another problem. Besides, when modeling emission from graphene one should take into consideration the peculiarities of electron energy spectra [17].

\section{Acknowledgements}

The authors are thankful to D.A. Zenyuk for assistance in preparing graphical illustrations.

The work was supported by Russian Foundation for Basic Research (Project No. 12-01-00339).

\section{REFERENCES}

[1] Fursey, G. N. Field Emission in Vacuum Microelectronics, Springer, US. 2005.

[2] Marrese C. A. A review of field emission cathode technologies for electric propulsion systems and instruments. IEEE Aerospace Conference Proceedings, 2000, Big Sky, Montana, vol.4, 85-98, 2000.

[3] Koike, A, Fujino, T., Neo, Y., Mimura,., Yoshida, T., Nishi, T., Nagao, M., Murata, H. A functional tiny electron gun for a true microcolumn. Technical digest of the 24th International Vacuum Nanoelectronics Conference, Historische Stadthalle Wuppertal, Germany, 35 - 36, 2011.

[4] Peng Liu, Yang Wei, Kai Liu, Liang Liu, Kaili Jiang, and Shoushan Fan. New-Type Planar Field Emission Display with Superaligned Carbon Nanotube Yarn Emitter, Nano Lett., 12, 2391-2396, 2012.

[5] Smith, B.C., Hunt, Charles E., McLeod, C. A. A field-emission monochromatic micro-x-ray source, Technical digest of the 24th International Vacuum Nanoelectronics Conference, Historische Stadthalle Wuppertal, Germany, 111-112, 2011.

[6] Schreiner, R., Fac. Dams, F., Prommesberger, C., Bornmann, B. Silicon-based integrated field emission electron sources for sensor application, Technical digest of the 24th International Vacuum Nanoelectronics Conference, Historische Stadthalle Wuppertal, Germany, 19-20, 2011.

[7] Koops, H.W.P.; Al-Mudhafar, A.; Hartnagel, Hans L. Miniaturized THz source with free-electron beams, Technical digest of the 24th International Vacuum Nanoelectronics Conference, Historische Stadthalle Wuppertal, Germany, 187-188, 2011.

[8] Yuan-Ming Chang, Pin-Hsu Kao, Hung-Ming Tai, Hau-Wei Wang, Chih-Ming Lin, Hsin-Yi Lee and Jenh-Yih Juang. 
Enhanced field emission characteristics in metal-coated Si-nanocones Phys.Chem. Chem. Phys., v. 15, 10761, 2013.

[9] Goki Eda, H. Emrah Unalan, Nalin Rupesinghe, Gehan A. Amaratunga, and Manish Chhowalla. Field emission from graphene based composite thin films, Appl. Phys. Lett. Vol. 93, 233502, 2008.

[10] Zhong-Shuai Wu, Songfeng Pei, Wencai Ren, Daiming Tang, Libo Gao, Bilu Liu, Feng Li, Chang Liu, and Hui-Ming Cheng. Field Emission of Single-Layer Graphene Films Prepared by Electrophoretic Deposition, Adv. Mater., Vol. 21, 1756-1760, 2009.

[11] U. A. Palnitkar, Ranjit V. Kashid, Mahendra A. More, Dilip S. Joag, L. S. Panchakarla, and C. N. R. Rao. Remarkably low turn-on field emission in undoped, nitrogen-doped, and boron-doped graphene, Appl. Phys. Lett. Vol. 97, 063102, 2010.

[12] R. H. Fowler and L. Nordheim. Electron Emission in Intense Electric Fields, Proc. R. Soc. Lond. A, Vol. 119, 173-181,
1928.

[13] V.A. Fedirko, V.V. Guziy. An analytical model for the electric field of a wedge field emitter, Technical Digest of the 19th International Vacuum Nanoelectronics Conference (Guilin, China), IEEE, 247-248, 2006.

[14] L.D.Landau, E.M. Lifshitz. Electrodynamics of Continuous Media, Pergamon Press, London, 1960.

[15] V. A. Fedirko, S. V. Polyakov, and D. A. Zenyuk. Matrix Method for Simulation the Tunneling Transfer, Mathematical Models and Computer Simulation, v 2 No. 6 , pp 704-713, 2010.

[16] V. A. Fedirko, S. V. Polyakov, and D. A. Zenyuk. Transformation Matrix Method for Tunnel Effect Simulation, Physics of Particles and Nuclei Letters, Vol. 8, No. 5, pp. 463-466, 2011.

[17] A. K. Geim, K. S. Novoselov. The rise of graphene, Nature Materials, Vol. 6, 183-191, 2007. 